\title{
Force between two parallel screw dislocations and application to linear screw dislocation pileups - Gauge theory results
}

\author{
M C VALSAKUMAR, DEBENDRANATHSAHOO* and S KANMANI \\ Materials Science Division, Indira Gandhi Centre for Atomic Research, Kalpakkam 603102 , \\ India

\begin{abstract}
An analytic expression for the force between two parallel screw dislocations, derived earlier on the basis of the gauge theory of dislocations, has been used to investigate the static distribution of a given number $N$ of parallel screw dislocations confined between two immobile dislocation obstacles. It is shown that in the limit of a continuous distribution of dislocations the equilibrium condition leads to a Fredholm integral equation of first type which does not admit any nontrivial solution. Implication of this result is discussed. For a finite number of dislocations, the ratio $(\eta)$ of the obstacle separation to the core radius is an important parameter governing the nature of solution of the discrete equation. It is found that
\end{abstract} \\ for a given $N$, there is a critical value $\eta_{\mathrm{c}}$ below which there does not exist any solution.
}

Keywords. Dislocation; gauge theory; interaction; force; screw dislocation; pileup; chaos.

\section{Introduction}

Behaviour of a group of dislocations confined between two obstacles in a material medium is an interesting problem. Much work has been done on these so-called dislocation pileups using the classical elasticity theory (Eshelby et al 1951; Bilby and Eshelby 1968; Hirth and Lothe 1968). The classic paper in this area is that of Eshelby et al (1951). It is also a well known fact that classical elasticity is inapplicable inside the core region of a dislocation. The stress field becomes singular when the classical expression is extrapolated to the dislocation line position. When two dislocations come near one another within a distance of their core radius, classical elasticity offers no means of analyzing their interaction. Since the ensemble of $N$ dislocations is analyzed by superposing the two-body interaction forces, a better analysis of the problem is clearly warranted. It was shown earlier (Valsakumar and Sahoo 1988) that the gauge theory of dislocations proposed by Kadic and Edelen (1983) admits a physically meaningful solution for the stress field of a screw dislocation which agrees with the stress field expression of the classical elasticity theory at large distances and is finite inside the core region. Later an analytic expression was obtained for the interaction force between two parallel dislocations (Valsakumar and Sahoo 1997). It is the purpose of this work to reinvestigate the dislocation pileup problem in the light of this new result.

In the following section, we summarize the key concepts and steps in the gauge theory of dislocations. Next in $\S 3$, we formulate the pileup problem and derive the main results of this work. Finally in $\S 4$, we conclude with a brief discussion on the implications of our results.

\section{Summary of the gauge theory of dislocations}

In the gauge approach, one starts with the Lagrangian density of the perfect elastic continuum

$$
\mathscr{L}_{0}=-\frac{1}{2}\left[\lambda\left(e_{i i}\right)^{2}+2 \mu e_{i j} e_{i j}\right],
$$

\footnotetext{
*Author for correspondence
} 
where $e_{i j}=\frac{1}{2}\left(u_{i, j}+u_{j, i}\right)$ is the strain tensor, $u_{i}$ the elastic displacement field $(i, j=x, y, z)$, and $u_{i, j}=\partial u_{i} / \partial x_{j} . \mathscr{L}_{0}$ is invariant under the transformation $u_{i} \rightarrow u_{i}+c_{i}$, where $c_{i}$ 's are constants. Thus it has $T(3)$ global gauge symmetry - an internal symmetry (since $x_{i}$ 's remain unchanged). When $c_{i}$ 's are made $\vec{x}$ dependent, the above invariance is lost. Restoration of local gauge invariance is possible if new fields $\phi_{i j}$, the gauge potential fields, are introduced and the definition of derivative is changed as below:

$$
u_{i, j} \rightarrow u_{i, j}+\phi_{i j}
$$

Under $u_{i} \rightarrow u_{i}+c_{i}(\vec{x})$,

$$
\phi_{i j} \rightarrow \phi_{i j}^{\prime}=\phi_{i j}-c(x)_{i, j} \text {. }
$$

This means $e_{i j}$ is to be replaced by

$$
E_{i j}=e_{i j}+\frac{1}{2}\left(\phi_{i j}+\phi_{j i}\right) \text {. }
$$

The Lagrangian $\mathscr{L}_{0}\left(e_{i j}\right) \rightarrow \mathscr{L}_{0}\left(E_{i j}\right)$ def $=\tilde{\mathscr{L}}_{0}$ maintains the local gauge symmetry. The Lagrangian corresponding to the $\phi_{i j}$ fields is

$$
\mathscr{L}_{1}=-\frac{s}{2} F_{i j}^{a} F_{i j}^{a}, F_{i j}^{a}=\phi_{a j, i}-\phi_{a i, j},
$$

is the dual of the dislocation density tensor $\alpha_{i j}=\varepsilon_{i k l} \phi_{j l, k}$. The total Lagrangian density of the system is $\mathscr{L}=\widetilde{\mathscr{L}}_{0}+\mathscr{L}_{1}$. This leads to coupled partial differential equations for $u_{i}, \phi_{i j}$. It is possible to obtain a solution having the symmetry of classical screw dislocation. It's dislocation density tensor and the displacement field are respectively

$$
\alpha_{i j}=\delta_{i 3} \delta_{j 3} \alpha(\rho), \quad \alpha(\rho)=\frac{\kappa^{2} b(\infty)}{2 \pi} K_{0}(\kappa \rho), u_{i}=\delta_{i 3} u_{3}(\rho) .
$$

Here $\rho=\sqrt{x^{2}+y^{2}}$ is the radial coordinate and $\kappa=\sqrt{\mu / s}$ and $b(\infty)$ is the asymptotic value of the 'Burgers vector'.

$$
b(\rho)=b(\infty)\left[1-\kappa \rho K_{1}(\kappa \rho)\right] .
$$

$K_{n}$ is the modified Bessel function of second kind and order $n$. The stress field of the above dislocation is more physical than the classical solution:

$$
\begin{aligned}
& \sigma_{31}=\sigma_{13}=-\frac{\mu b(\infty)}{2 \pi \rho} \sin \theta\left[1-\kappa \rho K_{1}(\kappa \rho)\right], \\
& \sigma_{32}=\sigma_{23}=+\frac{\mu b(\infty)}{2 \pi \rho} \cos \theta\left[1-\kappa \rho K_{1}(\kappa \rho)\right] .
\end{aligned}
$$

Note that (2) has asymptotic agreement with classical stress expression at large distances and that the 'core' of the dislocation emerges naturally through the parameter $\kappa$ (core radius $\sim \kappa^{-1}$ ). Next consider the interaction between two screw dislocations using gauge theoretic approach. Apply this result to reanalyse the classic result of Bilby and Eshelby (1968) on the distribution of linear dislocation arrays confined between two obstacles. Superposing solutions of two parallel dislocations-one at the origin and the other at $\vec{R}=(X, Y)$, we get

$$
\begin{aligned}
\phi_{31}(\vec{\rho})= & -\frac{b_{1} y}{2 \pi \rho^{2}}\left(1-\kappa \rho K_{1}(\kappa \rho)\right) \\
& +\frac{b_{2}(Y-y)}{2 \pi \rho^{\prime 2}}\left(1-\kappa \rho^{\prime} K_{1}\left(\kappa \rho^{\prime}\right)\right),
\end{aligned}
$$


where $\rho^{\prime}=|\vec{R}-\vec{\rho}|$. The potential energy of these two dislocations are calculated and the interaction term $U_{12}(\vec{R})$ depending only on $\vec{R}$ is obtained. From $U_{12}$, we obtain $\vec{F}(\vec{R}, \kappa)$ - the force acting on dislocation 1 due to dislocation 2 :

$$
\begin{aligned}
\vec{F}(\vec{R}, \kappa) & =-\left[\frac{\partial U_{12}(\vec{\rho})}{\partial \rho}\right]_{\rho=R} \hat{R} \\
& =\frac{\mu b_{1} b_{2}}{2 \pi R}\left[1-\kappa R K_{1}(\kappa R)\right] \hat{R} \equiv \vec{F}^{\mathrm{cl}}(\vec{R})\left[1-\kappa R K_{1}(\kappa R)\right] .
\end{aligned}
$$

Note that $\vec{F}$ is finite inside the core and that it agrees with the classical force $\vec{F}^{\text {el }}$ asymptotically.

\section{The pileup problem}

Consider $N$ parallel screw dislocations of same sign in a line segment of length $2 l$ between two obstacles at $\pm l$. The question arises as to what are the equilibrium positions $X_{i}=l x_{i}\left(-1 \leqslant x_{i} \leqslant+1\right)$ of these dislocations? Using the fact that the net force on any dislocation must be zero, one gets

$$
\sum_{k \neq j} F_{j, k}+F_{0}\left(x_{j}\right)=0, j=1, \ldots, N,
$$

where $F_{j, k}$ is the force on the $j$ th dislocation at $x_{j}$ due to the $k$ th one at $x_{k}$ and $F_{0}\left(x_{j}\right)$ the force on the $j$ th dislocation due to the obstacles.

\subsection{Classical analysis}

According to the analysis based on classical elasticity theory, when $F_{0}$ is a rational function, the above equation can be converted into a differential equation for a function $g(x)$ whose zeros give the required result. For simplicity consider the case $N=2$ and take the obstacles also to be dislocations of the same type. Classical force balance condition on dislocations at $x_{1}$ and $x_{2}$ give

$$
\begin{aligned}
& \frac{1}{x_{1}-x_{2}}+\frac{1}{x_{1}-1}+\frac{1}{x_{1}+1}=0, \\
& \frac{1}{x_{2}-x_{1}}+\frac{1}{x_{2}-1}+\frac{1}{x_{2}+1}=0 .
\end{aligned}
$$

$x_{1}$ and $x_{2}$ are given by the zeros $\pm 1 / \sqrt{5}$ of $\mathrm{d} P_{3}(x) / \mathrm{d} x\left(P_{n}(x)=\right.$ Legendre polynomial of order $n$ ). For the general case of $N$ dislocations between two fixed ones, the equilibrium positions are given by the $N$ zeros of $\mathrm{d} P_{N+1}(x) / \mathrm{d} x$. When $N$ is large, it is convenient to have a description of the positions in terms of the number density $\rho(x)$ (per unit length) of dislocations (this is different from the dislocation density tensor used earlier). Then the force balance equation becomes

$$
\rho(x)\left[\int_{-1}^{+1} \mathrm{~d} y \rho(y) F^{\mathrm{cl}}(x-y)+F_{0}(x)\right]=0 .
$$


This is a Fredholm integral equation of the first kind with a singular kernel and the solution is given by

$$
\rho(x)=\frac{N}{\pi \sqrt{1-x^{2}}}+\frac{1}{\pi^{2}} \int_{-1}^{+1} \mathrm{~d} y \sqrt{\frac{1-y^{2}}{1-x^{2}}} \cdot \frac{F_{0}(y)}{x-y} .
$$

\subsection{Gauge theoretic analysis}

To perform the gauge theoretic analysis make the replacement

$$
\frac{1}{x_{k}-x_{l}} \rightarrow \frac{1-\eta\left|x_{k}-x_{l}\right| K_{1}\left(\eta\left|x_{k}-x_{l}\right|\right)}{x_{k}-x_{l}}
$$

i.e. $F^{\mathrm{cl}} \rightarrow F(x, \eta)$, where $\eta=\kappa l$ ( note that $\eta \rightarrow \infty$ gives the classical result). The force balance equations are now transcendental and therefore Eshelby's trick cannot be used for solving them. Consider first the 2-dislocation 'pileup'. When $\eta$ is below a critical value $\eta_{\mathrm{c}}(2) \approx 3 \cdot 15$, no solution for $x_{1}$ and $x_{2}$ is possible in the interval $[-1,1]$. This value of $\eta$ corresponds roughly to the case of the cores of the adjacent dislocations just touching one another. In general, for a given $N$, there exists an $\eta_{\mathrm{c}}(N)$ below which no stable solution is possible. That is, when the number of dislocations in between the obstacles exceeds a critical value, the system becomes unstable. Now consider the case of large $N$, the force balance equation has one trivial solution $\rho(x)=N \delta(x)$ corresponding to all the mobile dislocations being at the same place. This solution is unstable and agrees with the intuitive idea that all dislocations cannot reside at the same place. Is there a nontrivial solution? We can show that there is no continuous solution which is nonnegative definite and normalizable for any finite $\eta$. The proof is given in the appendix. This implies that the dislocation with a Burgers vector $b$ cannot be described in terms of a distribution of dislocations with infinitesimal Burgers vector. That is, the dislocations must necessarily be treated as discrete objects.

\section{Conclusions}

Gauge theory gives a force between two parallel dislocations (i) that agrees with the continuum elasticity solution at large separation and (ii) that vanishes at the origin. Two dislocations can 'pass through' each other. If the number of dislocations in between two obstacles exceeds a limit, the system becomes unstable. If the confining forces are not sufficiently strong, the dislocations will move out of the region. Otherwise they will execute chaotic motion. The dislocations must necessarily be treated as discrete objects.

\section{Acknowledgement}

We had long association with our esteemed colleague Dr K P Gopinathan who has recently retired. We dedicate this work to him. 


\section{Appendix}

Consider the integral equation

$$
\left[\int_{-1}^{+1} \mathrm{~d} y \rho(y) F(x-y, \eta)+F(x-1, \eta)+F(x+1, \eta)\right]=0, \quad x \in[-1,+1]
$$

corresponding to two identical dislocations at $x= \pm 1$ acting as obstacles. Here $F(x, \eta)=\eta f(\eta x)$ and $f(u)=\left(1-|u| K_{1}(|u|)\right) / u$. Note that $f(-u)=-f(u)$ and in the limit $u \rightarrow 0$ and $u \rightarrow \infty, f(u) \rightarrow 0$. Using the integral representation

$$
K_{1}(u)=u \int_{1}^{\infty} \mathrm{d} t \exp (-u t) \sqrt{t^{2}-1},
$$

one may write

$$
f(u)=\frac{1}{u}\left[\int_{0}^{u} \mathrm{~d} v v \exp (-v)+\int_{u}^{\infty} \mathrm{d} v \exp (-v)\left(v-\sqrt{\left(v^{2}-u^{2}\right)}\right)\right] ; u>0,
$$

which implies that $f(u)>0$ and finite for $0<u<\infty$. Clearly the kernel in (7) is nonsingular. Also note that except for $u=u_{*}$ where $f(u)$ is maximum, there are two values of $u$ (say $u$ and $u^{\prime}$ ) for which $f(u)$ has the same value. Let $u_{m}(u)$ denote the minimum of these

$$
u_{m}(u)=\min \left(u, u^{\prime}\right), f(u)=f\left(u^{\prime}\right) .
$$

Now consider (7) for $x=-1+\varepsilon$ and $x=1-\varepsilon$. Subtracting the resulting equations, we obtain yet another integral equation for $\rho$.

where

$$
\int_{-1}^{+1} \mathrm{~d} y \rho(y) g(\varepsilon, y, \eta)+h(\varepsilon, \eta)=0,
$$

$$
\begin{gathered}
g(\varepsilon, y, \eta)=F(1-\varepsilon-y, \eta)+F(1-\varepsilon+y, \eta) \\
h(\varepsilon, \eta)=2[F(2-\varepsilon, \eta)-F(\varepsilon, \eta)] .
\end{gathered}
$$

Define $\varepsilon_{*}=\min \left(\varepsilon_{1 *}, \varepsilon_{2 *}\right)$, where $\varepsilon_{1 *}=u_{m}\left(2-\varepsilon_{1 *}\right)$, and $\varepsilon_{2 *}=u_{m}\left(2-\varepsilon_{1 *}\right)$. It is easy to see that both $g(\varepsilon, y, \eta)$ and $h(\varepsilon, \eta)$ are positive for $0<\varepsilon<\varepsilon_{*}$. Thus $\rho(y)$ has to be negative for some $y$ for the auxiliary integral equation (8) to be satisfied. Therefore (7) does not allow for a positive definite solution.

\section{References}

Bilby B A and Eshelby J D 1968 Dislocations and the theory of fracture, in F racture: An advanced treatise (ed.) H Liebowitz (New York: Academic Press) Vol. 1 p. 100

Eshelby J D, Frank F C and Nabarro F R N 1951 Philos. Mag. 42351

Hirth J P and Lothe J 1968 Theory of dislocations (New York: McGraw Hill) Ch. 21

Kadic A and Edelen D G B 1983 A gauge theory of dislocations and disclinations (Berlin: Springer Verlag) Valsakumar M C and Sahoo D 1988 Bull. Mater. Sci. 103

Valsakumar M C and Sahoo D 1996 Phys. Lett. A215 305 